\title{
Paraneoplastic Neurological Syndrome Presenting as New-Onset Refractory Status Epilepticus
}

\author{
Amanda Nayberg, PA' - Linda Liu, PA' - Tanya Kappor-Maini, DO² - David Ledoux, MD²
}

A 78-year-old man presented to our emergency department with generalized tonic-clonic seizures of unknown duration and fever of $38.6{ }^{\circ} \mathrm{C}$.

\section{History}

He had a medical history significant for ischemic cardiomyopathy, prostate cancer in remission status post chemotherapy, vocal cord cancer status post radiation, and chronic renal insufficiency. Further history was provided by the patient's wife, who denied alterations in her husband's mood, memory, or affect but reported alteration in taste when eating, general malaise, gastrointestinal disturbance, and weakness.

\section{Physical examination}

The patient was oriented to self and date and was able to follow simple commands. He was hemodynamically stable, except for a fever of $38.6^{\circ} \mathrm{C}$. A bolus of valproate sodium, $1000 \mathrm{mg}$ was administered, and a standing intravenous (IV) valproate sodium, $500 \mathrm{mg}$ twice a day was started.

\section{Diagnostic Testing}

Computed tomography (CT) scans

of the brain were conducted, results of which did not show hemorrhage or infarct. A lumbar puncture was performed, results of which showed an elevated protein level of $0.059 \mathrm{~g} / \mathrm{dL}$. Empiric antibiotics were discontinued after cerebral spinal fluid (CSF) and blood culture results were negative for microorganisms. Magnetic resonance imaging (MRI) scans of the brain were not conducted because of a noncompatible implantable pacemaker.

On hospital day 2, the patient was noted to be unresponsive with a left gaze preference and left hemiparesis with noxious stimuli. He was given lorazepam, $2 \mathrm{mg}$ with a loading dose of levetiracetam, $3000 \mathrm{mg}$ and was administered levetiracetam, $1500 \mathrm{mg}$ twice daily.

A continuous electroencephalography (cEEG) was started, which initially showed abundant right hemispheric lateralized periodic discharges every 0.5 to 1.0 seconds, concerning for nonclinical

\section{AFFILIATIONS:}

'Physician Assistant, Neurosurgery/Neurocritical Care, Northwell Health South Shore University Hospital, Bayshore, New York

${ }^{2}$ Neurology Department, Northwell Health South Shore University Hospital, Bayshore, New York ${ }^{3}$ Neurocritical Care Department, Northwell Health South Shore University Hospital, Bayshore, New York

\section{CITATION:}

Nayberg A, Liu L, Kapoor-Maini T, Ledoux D. Paraneoplastic neurological syndrome presenting as new-onset refractory status epilepticus. Consultant. Published online February 16, 2022. 10.25270/ con.2022.02.00005

Received June 28, 2021. Accepted August 30, 2021.

\section{DISCLOSURES:}

The authors report no relevant financial relationships.

\section{CORRESPONDENCE:}

Amanda Nayberg, PA, South Shore University Hospital, 301 East Main Street, Bay Shore, NY 11706 (schill9@gmail.com)

status epilepticus. The differential diagnosis included stroke and encephalitis. Later that afternoon, cEEG results showed some improvement after IV lorazepam was administered, but right hemispheric lateralized periodic discharges then occurred every 1 to $2 \mathrm{~Hz}$, for which fosphenytoin was added to the treatment regimen. The subsequent cEEG was consistent with nonconvulsive status epilepticus that spread to the left hemisphere. Antiepileptic therapy was maximized, and the patient was intubated and started on a propofol infusion, requiring transfer to the intensive care unit (ICU).

CT noncontrast and CT angiography scans of the brain were performed, results of which showed no evidence of acute infarct, intracerebral hemorrhage, mass lesion, mass effect, large vessel occlusion, or aneurysm. However, a CT perfusion scan of the brain showed a focus of increased perfusion without evidence of core infarct in the left temporoparietal region. In addition, an cEEG recorded on the same day showed right temporal seizure focus with improvement of nonconvulsive status epilepticus. Because no evidence of infectious, structural, or metabolic etiologies were noted, neoplastic or autoimmune encephalitis was high on the differential diagnosis. Further CSF studies were conducted, including evaluating for anti-Hu, anti-amphiphysin, anti-CV2, and anti-collapsin response mediator protein 5 (CRMP5). Empirical methylprednisolone, $1000 \mathrm{mg}$ daily was started for 5 days.

On hospital day 3 , findings from a neurologic examination showed improvement, the $c E E G$ showed no further evidence of seizures, and criteria was 
met for extubation. The next day, the patient remained awake and alert and followed commands with improvement in his neurologic deficits. Ultimately, the patient no longer had critical care needs and was downgraded to the medical floor with consistently improved neurologic examination findings. The CSF studies were positive for anti-Hu, consistent with neurological autoimmunity, supporting a diagnosis of paraneoplastic autoimmune syndrome. A CT scan of the chest, abdomen, and pelvis conducted upon admission did not reveal any masses or evidence of small-cell lung cancer. Of note, the patient's prostate-specific antigen level was tested, which was low at $0.22 \mathrm{ng} / \mathrm{mL}$.

One day after completing a 5-day course of solumedrol, the patient was noted to be obtunded, hypotensive, and hypoxic on the medical floor. He was reintubated, started on vasopressors, pan-cultured, and returned to the ICU. Despite aggressive resuscitation with fluids, vasopressors, and stress-dose steroids, the patient died less than 24 hours after returning to the ICU. Postmortem cultures of his sputum and blood grew methicillin-resistant Staphylococcus aureus (MRSA).

\section{Discussion}

New-onset refractory status epilepticus (NORSE) is a serious condition that requires prompt cessation of seizures with investigation and treatment of the underlying cause. Without a clear, active, structural, toxic, metabolic, infectious, or autoimmune etiology, paraneoplastic autoimmune syndrome should become high on the differential diagnosis. Despite a history of or current malignancy, paraneoplastic syndrome should still be considered, because a subgroup of paraneoplastic disorders can present with cancer that has resolved or can present within 5 years of diagnosis of cancer. During a paraneoplastic syndrome workup, if anti-Hu antibody is present, prostate cancer should be considered as a potential etiology. This case report

\section{About $20 \%$ of patients with refractory status epilepticus may also have NORSE. The syndrome can present with a prodromal illness 1 to 14 days prior to development of seizures. The symptoms may include upper respiratory tract infections, gastroenteritis, fever, fatigue, or confusion.}

describes a patient with a rare cause of paraneoplastic syndrome with an atypical clinical representation.

This was an interesting case of a patient who presented with fever, NORSE, and a rare secondary cause of paraneoplastic syndrome. Our patient's continuous seizure activity, despite treatment with an adequate dose of benzodiazepines and second-line IV antiepileptic medications, supports the definition of NORSE. ${ }^{2}$ Briefly, cryptogenic NORSE is a clinical presentation of acute refractory status epilepticus that does not have a clear, active, structural, toxic, metabolic, infectious, or autoimmune etiology in $50 \%$ of patients and has a mortality rate of $16 \%$ to $27 \% .^{3}$ A subcategory of NORSE, called febrile infection-related epilepsy syndrome (FIRES), requires a febrile infection between 2 weeks and 24 hours prior to refractory status epilepticus in order to be diagnosed. ${ }^{2}$ The most common etiologies for this subcategory are autoimmune, paraneoplastic encephalitis, or an infectious source. ${ }^{2}$

Our patient presented with gastrointestinal symptoms, fever, general malaise, and weakness, which were broadly covered with antibacterial and viral medications, although central nervous system, pulmonary, gastrointestinal, urologic, or bloodstream sources of infection were inconclusive. A diagnostic evaluation with no significant pathology was reported on a CT scan of the brain, chest, abdomen, and pelvis. Ideally, an MRI of the brain with and without contrast is useful to identify any structural or focal abnormalities, along with symmetrical or asymmetrical hyperintensity on T2/FLAIR sequences within the limbic or neocortical regions. However, our patient's cardiac defibrillator was not compatible with an MRI scan.

About $20 \%$ of patients with refractory status epilepticus may also have NORSE. ${ }^{4}$ The syndrome can present with a prodromal illness 1 to 14 days prior to development of seizures. The symptoms may include upper respiratory tract infections, gastroenteritis, fever, fatigue, or confusion. ${ }^{2-4}$ The most common type of seizure is focal motor that can transition into tonic-clinic seizures. Other seizure types are focal seizures associated with impaired awareness or myoclonic seizures. ${ }^{5}$

The etiology of NORSE may be unknown because of limitations in the awareness of this disease, its broadening definition, and its comprehensive diagnostic evaluation. The initial evaluation of a patient with refractory status epilepticus should include a comprehensive metabolic profile examining for magnesium, calcium, and phosphorus levels; a complete blood cell count; urine or blood toxicology screenings; erythrocyte sedimentation rate; and C-reactive protein level. Neuroimaging such as CT scans and/or MRI with and without contrast of the brain should be conducted to rule out structural sources. ${ }^{6} \mathrm{~A}$ lumbar puncture becomes essential if the etiology of 
refractory seizures is unclear. ${ }^{6}$

As with our patient, a lumbar puncture was required for comprehensive CSF testing to evaluate for the 2 most common causes of NORSE-nonparaneoplastic or paraneoplastic autoimmune encephalitis-since the laboratory results were inconclusive. Autoimmune encephalitis encompasses several diseases and pathophysiologies. The most common nonparaneoplastic autoimmune encephalitis antibodies are anti-Nmethyl-D-aspartate receptor (NMDAR), anti-voltage-gated potassium channels (anti-VGKC), anticontactin-associated protein-like 2 (anti-CASPR2), anti-GABA, anti-glycine, and anti-GAD65. Another common encephalopathy is a steroid-responsive encephalopathy associated with autoimmune thyroiditis (Hashimoto encephalitis). ${ }^{7}$ There may be clues to various causes of these diseases, yet beyond taking a history or conducting a physical examination, limited testing may yield inconclusive results. A broadened approach to testing, such as we did with our patient after ruling out an infectious source, may lead to the correct diagnosis.

There are 3 groups of autoimmune disorders associated with a different pathophysiology attributing to paraneooplastic neurologic syndromes (PNS). The first group is associated with antibodies to intracellular antigens, which are the classic paraneoplastic disorders, and involves T-cell response-targeting neurons. ${ }^{8}$ The onconeural antigens, which are expressed through gene amplification, protein overexpression, or tumor genetic alterations, activate $B$ cells that yield antibodies that target the intracellular antigens via the cytotoxic T cell. The paraneoplastic antibodies are not directly pathogenic. The antibodies in this group are pathogenic, unlike those in group 1 , and the cancer associations are variable. The second group are antibodies against proteins of intracellular sites of the GABAergic and glycinergic receptor. The third group are associated with antibodies against cell surface synaptic antigens. ${ }^{8}$

In the paraneoplastic encephalitis group, the most common antibodies are the anti-NMDAR, anti-CRMP5, anti-Ma2, anti-amphiphysin, and anti-VGKC. The anti-Hu antibody was the first type of onconeural antibody described, was identified in our patient, and is known to be molecularly associated with cancer. ${ }^{9}$ The association with small-cell lung cancer may be as high as $86 \%$ with a poor outcome. ${ }^{9}$ In a recent prospective observational study, the researchers reviewed case histories from 37 patients with paraneoplastic neurologic syndrome identified via the Euronetwork and PubMed databases. ${ }^{9}$ The classical paraneoplastic neurologic syndromes described in the study were paraneoplastic encephalitis, limbic encephalitis, paraneoplastic neurologic syndrome cerebellar degeneration, subacute sensory neuronopathy, and gastrointestinal autonomia. The nonclassical paraneoplastic neurologic syndromes described in the remaining patients were either brainstem encephalitis or peripheral neuropathy. The anti-Hu antibody was detected in $50 \%$ of patients with paraneoplastic neurologic syndrome and prostate cancer. Paraneoplastic neurologic syndrome had occurred in $32 \%$ of these patients prior to the diagnosis of prostate cancer, with a time delay of less than 1 year in a majority of patients. ${ }^{9}$

Paraneoplastic neurologic syndromes are autoimmune phenomena classified into 3 subcategories: ${ }^{1}$

1. A classical syndrome including encephalomyelitis, limbic encephalitis, subacute cerebellar degeneration, and opsoclonus-myoclonus. These patients present with weeks to months of noted cognitive decline, personality changes, ataxia, cranial nerve deficits, weakness, or numbness, which was not consistent with our patient's neurologic manifestations.

2. A nonclassical syndrome that resolves with cancer treatment.

3. A nonclassical syndrome with paraneoplastic antibodies and cancer that develops within 5 years of neurological disturbance.

4. A neurological syndrome with paraneoplastic antibodies, but no cancer

Our patient did not have active malignancy, because the CT scan of his head, neck, chest, abdomen, and pelvis was not abnormal and his prostate-specific antigen level was within normal limits.

\section{Conclusions}

We diagnosed a unique presentation of a nonclassical paraneoplastic neurologic syndrome associated with a paraneoplastic antibody in a patient presenting with NORSE and, specifically, a subcategory of NORSE called FIRES that rapidly improved with high-dose corticosteroid treatment. Half the cases of FIRES are caused by autoimmune or paraneoplastic encephalitis. In two-thirds of patients with NORSE, most of the preceding febrile illnesses are described as mild, associated with malaise and fatigue, and associated with symptoms of upper respiratory or gastrointestinal illness, with which our patient presented. ${ }^{2,4}$ While our patient had no evidence of small-cell lung cancer, he was in remission from prostate cancer. While paraneoplastic neurologic syndrome is not typically associated with prostate cancer, it should be considered in men with a history of or suspicion for prostate cancer and with new-onset refractory seizures.

NORSE is relatively an uncommon clinical entity with no clear etiology in most cases. It is associated with significant mortality and long-term neurological sequelae, requiring a multidisciplinary approach to management. Existing evidence regarding the etiology, diagnostic workup, and treatment options are based on expert opinion and retrospective case series. General awareness about the nature of the disease and treatment options available should be promoted among medical professionals. Empiric treatment should be started while waiting for confirmatory results, as a delay in treatment results in worse outcomes. 


\section{References}

1. Graus F, Delattre JY, Antoine JC, et al. Recommended diagnostic criteria for paraneoplastic neurological syndromes. J Neurol Neurosurg Psychiatry. 2004;75(8):1135-1140. https://doi.org/10.1136/jnnp.2003.034447

2. Hirsch LJ, Gaspard N, van Baalen A, et al. Proposed consensus definitions for new-onset refractory status epilepticus (NORSE), febrile infection-related epilepsy syndrome (FIRES), and related conditions. Epilepsia. 2018;59(4):739-744. https://doi.org/10.1111/ epi.14016

3. Gaspard N, Foreman BP, Alvarez V, et al. New-onset refractory status epilepticus: etiology, clinical features, and outcome. Neurology. 2015;85(18):1604-1613. https:// doi.org/10.1212/wnl.0000000000001940

4. Gaspard N, Hirsch LJ, Sculier C, et al. New-onset refractory status epilepticus (NORSE) and febrile infection-related epilepsy syndrome (FIRES): state of the art and perspectives. Epilepsia. 2018;59(4):745-752. https://doi.org/10.1111/epi.14022
5. Wilder-Smith EP, Lim EC, Teoh HL, et al. The NORSE (new-onset refractory status epilepticus) syndrome: defining a disease entity. Ann Acad Med Singap. 2005;34(7):417-420. https://annals.edu.sg/ pdf/34VolNo7200508/V34N7p417.pdf

6. Sculier C, Gaspard N. New onset refractory status epilepticus (NORSE). Seizure. 2019;68:72-78.

https://doi.org/10.1016/j.seizure.2018.09.018

7. Dalmau J. Status epilepticus due to paraneoplastic and nonparaneoplastic encephalitides. Epilepsia. 2009;50 Suppl 12:58-60. https://doi.org/10.1111/ j.1528-1167.2009.02352.x

8. Storstein A, Raspotnig M, Vitaliani R, et al. Prostate cancer, $\mathrm{Hu}$ antibodies and paraneoplastic neurological syndromes. J Neurol. 2016;263(5):1001-1007. https://doi.org/10.1007/s00415-016-8090-7

9. Tanaka K, Kawamura M, Sakimura K, Kato N. Significance of autoantibodies in autoimmune encephalitis in relation to antigen localization: an outline of frequently reported autoantibodies with a non-systematic review. Int $\mathrm{J} \mathrm{Mol}$ Sci. 2020;21(14):4941. https://doi.org/10.3390/ ijms21144941 